\title{
Topical Probiotics in Dermatological Therapy and Skincare: A Concise Review
}

Katlein França

Received: October 27, 2020 / Published online: December 19, 2020

(C) The Author(s) 2020

\section{ABSTRACT}

Recent studies have produced an increasing body of evidence that the intestinal microbiome plays an essential role in modulating systemic inflammation and skin diseases. The gut microbiome influences and modulates the host immune system, enabling immune tolerance of environmental and dietary antigens and protecting against pathogens. Emerging scientific evidence has demonstrated that oral probiotics can help treat certain skin diseases, such as acne, atopic dermatitis, photoaging, psoriasis, and wound healing. The aim of this paper is to review the current scientific evidence on topical probiotics and their effects on dermatological diseases and skincare and to clarify if the application of exogenous probiotics could also have the same benefit as oral probiotics in promoting positive bacterial balance to treat dermatologic conditions.

Keywords: Acne; Atopic dermatitis; Dermatologic therapy; Skincare; Topical probiotics; Wound healing

K. França $(\bowtie)$

Dr. Phillip Frost Department of Dermatology and Cutaneous Surgery, University of Miami Miller School of Medicine, Miami, FL, USA

e-mail: k.franca@med.miami.edu

\section{Key Summary Points}

Over the past decade, there is an increased interest in oral and topical probiotics for skincare and treatment of skin diseases

This paper reviews the current scientific evidence on the use of topical probiotics for the treatment of acne, atopic dermatitis, photoaging, skin aging, psoriasis, and wound healing.

While new products are released in the market, research groups have been trying to determine their efficacy, mechanism of action, safety, and indications.

As science continues to explore the interesting gut-skin axis and the effects of the microbiome on the skin, new studies will clarify the role of topical probiotics on skin disorders and skincare.

\section{DIGITAL FEATURES}

This article is published with digital features, including a summary slide to facilitate understanding of the article. To view digital features for this article, go to https://doi.org/10.6084/ m9.figshare.13318013. 


\section{INTRODUCTION}

Probiotics are defined as "live microorganisms which, when administered in adequate amounts, confer a health benefit on the host." In 1900 Louis Pasteur first identified the microorganisms responsible for fermentation, subsequently bringing health to and promoting the longevity of Bulgarian rural people if they consumed fermented foods such as yogurt and the bacteria they contained. He suggested that "lactobacilli might counteract the putrefactive effects of gastrointestinal metabolism that contributed to illness and aging." [1] Hippocrates said, 'all disease begins in the gut,' and over 2000 years later, science continues exploring this concept [2]. Several basic science and clinical studies have demonstrated that the gut microbiome's influence extends to distant organs, such as the skin, through complex mechanisms involved in the gut-skin axis. The use of prebiotics, probiotics, and symbiotics has shown promising effects on the prevention and treatment of different inflammatory skin disorders, such as acne and atopic dermatitis [3].

Over the past decade, interest in the use of oral and topical probiotics for skincare and the treatment of skin diseases has been increasing. While new products are released in the market, research groups have been trying to determine their efficacy, mechanism of action, safety, and indications. Lee et al. explain that they have shown efficacy in a number of limited trials involving acne treatment, atopic dermatitis, and rosacea [4].

The U.S. Food and Drugs Administration (FDA) categorizes probiotics into different categories, such as dietary supplements, foods, food additives, cosmetics, or drugs, based on each individual product. However, there is no regulation for topical probiotics, and currently there are no topical probiotic products approved by the FDA, which shows a need to improve the regulations for these products [4].

The aim of this article is to review the current scientific evidence on topical probiotics and its effects on dermatological diseases and skincare and to clarify if exogenous probiotics applications could also have the same benefit as oral probiotics in promoting positive bacterial balance to treat dermatologic conditions.

This article is based on previously conducted studies and does not contain any new studies with human participants or animals performed by any of the authors.

\section{ACNE}

Acne is one of the most common skin diseases with a lifetime prevalence of approximately $85 \%$

It is caused by multiple factors, such as alteration of follicular keratinization, increased and altered sebum production under androgen control, follicular colonization by Propionibacterium acnes, and complex inflammatory mechanisms that involve both innate and acquired immunity [5].

There is scientific evidence pointing out that the interaction between the skin microbes and host immunity plays a significant role in thepathogenesis of acne. Propionibacterium acnes is commonly found in sebum-rich areas and participates in the skin's microbiome, interacting with other common microorganisms, such as Staphylococcus epidermidis, Streptococcus pyogenes, and Pseudomonas species, and its over-proliferation has long been thought to contribute to the development of acne [6]. The microbiota of patients with acne differs from patients without acne. Individuals with acne have a unique microbial signature, and the use of systemic antibiotics can modulate the microbiota, changing its composition and diversity, especially of $P$. acnes, which correlates with acne severity. Challenges in current acne treatment include compliance with topical treatments that can commonly disrupt the skin barrier, causing dryness and skin irritation. Park et al. noted that probiotics modify several factors in the pathophysiology of acne development and can potentially improve compliance as well [7]. The connection between gut microbiota and acne has attracted attention. Authors of emerging studies focusing on the gut-brainskin connection and the immunobiology of acne have hypothesized that stress influences gut microbiota, leading to skin inflammation 
and thereby triggering acne, and that diet modifications and probiotics could change the gut microbiota and improve acne [8].

In 1912, a study published in the Journal of Cutaneous Diseases first reported that "topical bacteriotherapy" could be helpful to treat different skin disorders, including acne [9].

The term "bacteriotherapy" is currently reemerging among the scientific community, and it encompasses a variety of bacterial products and application modalities involving isolated or diverse bacterial species. It can be used broadly to describe any use of bacteria or bacterial components for therapeutic benefits and includes not only probiotics but also different types of bacteria-derived enzymes and bacterial transplant $[9,10]$.

The benefits of applying lactic acid bacteria to skin continued to be investigated decades later by Di Marzio et al. who showed that Streptococcus thermophilus, which can be found in yogurts, could increase the production of ceramides when applied topically to the skin for 7 days [11]. Bowe et al. highlighted that this work was relevant to acne therapy, particularly when one considers that some of the ceramides (e.g., phytosphingosine) provide both antimicrobial activity against $P$. acnes and also provide anti-inflammatory activity [12]. The topical application of phytosphingosine has been shown to reduce papules and pustules on acne patients [13]. An in vitro study evaluated the symbiotic ability of probiotic bacteria and konjac glucomannan hydrolysates (GMH) to inhibit $P$. acnes growth. The authors found that different probiotic bacteria strains were able to inhibit the growth of this skin bacterium species and that the presence of the GMH prebiotic significantly enhanced the inhibition [14]. A lactic acid bacterial strain isolated from a human fecal specimen and identified as Enterococcus faecalis SL-5 showed antimicrobial activity against Gram-positive pathogens assayed, especially against $P$. acnes. A lotion containing $E$. faecalis significantly reduced pustules compared to placebo lotion. Kang et al. consider that the anti-P. acnes activity produced by $E$. faecalis SL-5 has a potential role in the treatment of acne and could serve as an alternative to topical antibiotics [15].
Another species that has shown a potential to inhibit P. acnes is Streptococcus salivarius, a prominent member of healthy humans' oral microbiota. In addition to possessing antimicrobial activity, it also acts as an immunomodulator inhibiting different inflammatory pathways $[16,17]$.

Lopes et al. performed a study to check the capability of different probiotic strains to adhere to human skin and their antimicrobial activity against selected pathogens. These authors also evaluated cell-free culture supernatants (CFCS) of probiotic strains for their ability to prevent the formation or destruction of an established biofilm by selected pathogens and to search for potential quorum-sensing antagonists. The authors found that antimicrobial activity of the CFCS of selected probiotic strains was observed towards Escherichia coli, $P$. acnes, and Pseudomonas aeruginosa; however, none was able to inhibit the growth of Staphylococcus aureus [18].

It is also important to note that probiotics applied topically can also serve as a protective shield by functioning as a competitive inhibitor of binding sites, thereby preventing colonization by other potential pathogens $[4,19]$.

\section{ATOPIC DERMATITIS}

Atopic dermatitis (AD) is a chronic inflammatory skin condition that can cause intense pruritus and scaly lesions. The current treatment of patients with $\mathrm{AD}$ includes repair and protection of the skin barrier with moisturizers, anti-inflammatory medications, antihistamines, biologics such as dupilumab, and topical or systemic antimicrobial therapies [20]. As these traditional treatment options might be insufficient for certain patients or are associated with side effects, other nonpharmacological treatments, such as probiotics, are being investigated to treat this skin condition $[18,21]$.

Di Marzio et al. investigated the effects of a cream containing Streptococcus thermophilus on the levels of ceramides in the stratum corneum of patients with $\mathrm{AD}$. A 2-week application of the cream, which contained a sonicated preparation of the lactic acid bacterium Streptococcus 
thermophilus, onto the forearm skin of 11 patients led to a significant and relevant increase in the amounts of skin ceramides, possibly due to the hydrolysis of sphingomyelin by the bacterial sphingomyelinase. The patients also showed improvement in terms of erythema, scaling, and pruritus [21].

A prospective, double-blind, placebo-controlled clinical study involving 75 volunteers with $\mathrm{AD}$ aged 6 to 70 years who were randomized to receive either a cream containing a 5\% lysate of the nonpathogenic bacteria Vitreoscilla filiformis or a vehicle cream for 30 days was conducted. The authors found that the group receiving the $V$. filiformis lysate cream showed a significant improvement in their AD symptoms, possibly partly due to the reduction of Staphylococcus aureus but more likely related to a direct immunomodulatory effect on skin-associated immune responses [22]. It is known that $S$. aureus is predominant in the skin microbiota in patients with $\mathrm{AD}$ at bacterial loads correlating with disease severity.

The effect of a lotion containing the heattreated probiotic strain Lactobacillus johnsonii NCC 533 on Staphylococcus aureus colonization in 31 individuals with AD has also been investigated. The authors found that applying the lotion for 3 weeks controlled $S$. aureus colonization and was associated with local clinical improvement according to SCORAD (SCORing Atopic Dermatitis tool) [23].

A trial investigating the use of Roseomonas mucosa for AD treatment involved ten adult and five pediatric patients. Treatment with $R$. mucosa was associated with significant decreases in disease severity, topical steroid requirement, and $S$. aureus burden with no adverse effects or complications. The early results of this study support continued evaluation of $R$. mucosa therapy with a placebo-controlled trial [24].

Although research on topical probiotics for $\mathrm{AD}$ remains in its early stages, many trials so far have shown positive results.

\section{PHOTOAGING AND SKIN AGING}

One of the latest skincare trends for fighting photoaging and skin aging is probiotic skincare.
Although research on this subject is still in initial stages, new products are being launched onto the market at speed.

Initial studies in animal models and human clinical trials show that these products can slow the intrinsic and extrinsic aging process. Sharma et al. explain that probiotics can restore acidic skin $\mathrm{pH}$, alleviate oxidative stress, attenuate photoaging, improve skin barrier function, and enhance hair quality [25].

Ultraviolet exposure is an environmental factor that triggers abnormal production of reactive oxygen species and the expression of matrix metalloproteinases that are responsible for photoaging and skin aging. Plant extracts fermented with Lactobacillus buchneri (PELB), found in Kimchi, were evaluated regarding their anti-photoaging effects. The authors investigated the anti-photoaging effect of PELB in ultraviolet light (UVB)-induced photoaging in in vitro models. They pre-treated normal human dermal fibroblasts and epidermal keratinocytes with PELB and that had been exposed to UVB. They found that PELB decreased elastase activity and increased type I collagen expression in the UVB-induced photoaging in vitro model, reduced collagenase activity, and promoted the expression of moisture factor and antioxidant enzymes. The study results show that the PELB could be useful in the cosmetic industry due to its protective effects against UVB-induced photoaging [26]. Notay et al. investigated the use of topical Nitrosomonas eutropha for cosmetic improvement of facial wrinkles. They found a significant improvement in wrinkle depth severity, hyperpigmentation of the forehead and glabella in the group receiving high topical concentration of the probiotic formula [27].

\section{PSORIASIS}

Psoriasis is an immune-mediated, chronic inflammatory, genetic disease manifesting in the skin. Changes in the local microbiota have been implicated as a potential contributor to the disease as psoriatic lesions have shown a local microbiota distinct from that of healthy, unaffected skin [28]. The decrease of bacteria 
such as Staphylococcus epidermidis and P. acnes in the psoriatic skin microbiome may lead to higher Staphylococcus aureus colonization, causing cutaneous inflammation [29]. Firmicutes, Proteobacteria, Acidobacteria, Schlegelella, Streptococcaceae, Rhodobacteraceae, Campylobacteraceae, and Moraxellaceae species are present at higher levels in these patients, while Actinobacteria species are present at lower levels [30]. Group A $\beta$-hemolytic streptococcal infections are linked to guttate psoriasis [31].

Although data on probiotic supplementation in psoriasis treatment are limited, promising outcomes have been documented with oral probiotics interventions, and more studies are needed to explore the role of topical probiotics in psoriasis therapy [32].

\section{WOUND HEALING}

As wounds are associated with changes to local microflora due to injury and activation of the immune responses, topical probiotics have been investigated for infection prevention, inflammation regulation, and healing augmentation. They have been shown to be effective in a few studies involving human and animal models; however, there is significant variability among the studies [33].

The use of Lactobacillus plantarum as a potential therapeutic agent for the local treatment of Pseudomonas aeruginosa burn infections have shown positive results $[34,35]$. The same bacteria has been investigated for the treatment of non-diabetic and diabetic ulcers. Peral et al. found that it reduced bacterial load, neutrophils, and apoptotic and necrotic cells, modified interleukin- 8 production, and induced wound healing [36].

\section{FINAL CONSIDERATIONS}

Despite the promising results of the studies presented in this review, particularly on acne and atopic dermatitis, research on topical probiotics in skincare and dermatological therapy is still in the initial stages, and further studies are necessary to demonstrate their safety, efficacy, and the mechanism of action in different skin diseases. Potential side effects that need to be further investigated include allergic reactions to inactive ingredients, bacteremia, and antibiotic resistance transfer among pathogens [4]. Furthermore, there is a need for improved regulation and guidelines for product safety and use. As science continues to explore the interesting gut-skin axis and the effects of the microbiome on the skin, new studies will clarify the role of topical probiotics on different skin disorders skincare.

\section{ACKNOWLEDGEMENTS}

Funding. No funding or sponsorship was received for this study or publication of this article.

Authorship. The author meets the International Committee of Medical Journal Editors (ICMJE) criteria for authorship for this article, takes responsibility for the integrity of the work as a whole, and has given approval for this version to be published.

Disclosures. Katlein Franca is an Advisory Board Member of Dr. Brandt ${ }^{\circledR}$ Skincare and receives book royalties from the following publishers: Springer, Wiley-Blackwell, Novascience, and Juruá.

Compliance with Ethics Guidelines. This article is based on previously conducted studies and does not contain any new studies with human participants or animals performed by any of the authors.

Open Access. This article is licensed under a Creative Commons Attribution-NonCommercial 4.0 International License, which permits any non-commercial use, sharing, adaptation, distribution and reproduction in any medium or format, as long as you give appropriate credit to the original author(s) and the source, provide a link to the Creative Commons licence, and indicate if changes were made. The images or other third party material in this article are 
included in the article's Creative Commons licence, unless indicated otherwise in a credit line to the material. If material is not included in the article's Creative Commons licence and your intended use is not permitted by statutory regulation or exceeds the permitted use, you will need to obtain permission directly from the copyright holder. To view a copy of this licence, visit http://creativecommons.org/licenses/by$\mathrm{nc} / 4.0 /$.

\section{REFERENCES}

1. Gasbarrini G, Bonvicini F, Gramenzi A. Probiotics history. J. Clin. Gastroenterol. 2016;50:S116-9. https://doi.org/10.1097/MCG.0000000000000697.

2. van den Elsen LW, Poyntz HC, Weyrich LS, Young W, Forbes-Blom EE. Embracing the gut microbiota: the new frontier for inflammatory and infectious diseases. Clin Transl Immunol. 2017;6(1):e125. https://doi.org/10.1038/cti.2016.91.

3. Salem I, Ramser A, Isham N, Ghannoum MA. The gut microbiome as a major regulator of the gut-skin axis. Front Microbiol. 2018;9:1459. https://doi.org/ 10.3389/fmicb.2018.01459 (published 2018 Jul 10).

4. Lee GR, Maarouf M, Hendricks AJ, Lee DE, Shi VY. Topical probiotics: the unknowns behind their rising popularity. Dermatol Online J. 2019;25(5): 13030/qt2v83r5wk.

5. Tan AU, Schlosser BJ, Paller AS. A review of diagnosis and treatment of acne in adult female patients. Int J Womens Dermatol. 2017;4(2):56-71. https://doi.org/10.1016/j.ijwd.2017.10.006.

6. Platsidaki E, Dessinioti C. Recent advances in understanding Propionibacterium acnes (Cutibacterium acnes) in acne. F1000Res. 2018;7:F1000. https://doi.org/10.12688/f1000research.15659.1.

7. Park SY, Kim HS, Lee SH, Kim S. Characterization and analysis of the skin microbiota in acne: impact of systemic antibiotics. J Clin Med. 2020;9(1):168. https://doi.org/10.3390/jcm9010168.

8. Lee YB, Byun EJ, Kim HS. Potential role of the microbiome in acne: a comprehensive review. J Clin Med. 2019;8(7):987. https://doi.org/10.3390/ jcm8070987.

9. Peyri J. Topical bacteriotherapy of the skin. J Cutaneous Dis. 1912;30:688-9.
10. Hendricks AJ, Mills BW, Shi VY. Skin bacterial transplant in atopic dermatitis: knowns, unknowns and emerging trends. J Dermatol Sci. 2019;95(2): 56-61. https://doi.org/10.1016/j.jdermsci.2019.07. 001.

11. Di Marzio L, Cinque B, De Simone C, Cifone MG. Effect of the lactic acid bacterium Streptococcus thermophilus on ceramide levels in human keratinocytes in vitro and stratum corneum in vivo. J Investig Dermatol. 1999;113:98-106. https://doi. org/10.1046/j.1523-1747.1999.00633.x.

12. Bowe WP, Logan AC. Acne vulgaris, probiotics and the gut-brain-skin axis-back to the future? Gut Pathog. 2011;3(1):1. https://doi.org/10.1186/17574749-3-1.

13. Pavicic T, Wollenweber U, Farwick M, Korting HC. Anti-microbial and -inflammatory activity and efficacy of phytosphingosine: an in vitro and in vivo study addressing acne vulgaris. Int $\mathrm{J}$ Cosmet Sci. 2007;29:181-90. https://doi.org/10.1111/j. 1467-2494.2007.00378.x.

14. Al-Ghazzewi FH, Tester RF. Effect of konjac glucomannan hydrolysates and probiotics on the growth of the skin bacterium Propionibacterium acnes in vitro. Int J Cosmet Sci. 2010;32(2):139-42. https://doi.org/10.1111/j.1468-2494.2009.00555.x.

15. Kang BS, Seo JG, Lee GS, Kim JH, Kim SY, Han YW, et al. Antimicrobial activity of enterocins from Enterococcus faecalis SL-5 against Propionibacterium acnes, the causative agent in acne vulgaris, and its therapeutic effect. J Microbiol. 2009;47:101-9. https://doi.org/10.1007/s12275-008-0179-y.

16. Bowe WP, Filip JC, DiRienzo JM, Volgina A, Margolis DJ. Inhibition of Propionibacterium acnes by bacteriocin-like inhibitory substances (BLIS) produced by Streptococcus salivarius. J Drugs Dermatol. 2006;5:868-70.

17. Cosseau C, Devine DA, Dullaghan E, Gardy JL, Chikatamarla A, Gellatly S. et al. The commensal Streptococcus salivarius K12 downregulates the innate immune responses of human epithelial cells and promotes host-microbe homeostasis. Infect Immun. 2008;76:4163-75. https://doi.org/10.1128/ IAI.00188-08.

18. Lopes EG, Moreira DA, Gullón P, Gullón B, Cardelle-Cobas A, Tavaria FK. Topical application of probiotics in skin: adhesion, antimicrobial and antibiofilm in vitro assays. J Appl Microbiol. 2017;122(2):450-61. https://doi.org/10.1111/jam. 13349.

19. Brook I. Bacterial interference. Crit Rev Microbiol. 1999;25:155-72. 10408419991299211. 
20. Catherine Mack Correa M, Nebus J. Management of patients with atopic dermatitis: the role of emollient therapy. Dermatol Res Pract. 2012;2012: 836931. https://doi.org/10.1155/2012/836931.

21. Di Marzio L, Centi C, Cinque B, et al. Effect of the lactic acid bacterium Streptococcus thermophilus on stratum corneum ceramide levels and signs and symptoms of atopic dermatitis patients. Exp Dermatol. 2003;12:615-20.

22. Gueniche A, Knaudt B, Schuck E, et al. Effects of nonpathogenic Gram-negative bacterium Vitreoscilla filiformis lysate on atopic dermatitis: a prospective, randomized, double-blind, placebocontrolled clinical study. $\mathrm{Br} \mathrm{J}$ Dermatol. 2008;159(6):1357-63. https://doi.org/10.1111/j. 1365-2133.2008.08836.x.

23. Lanchet-Réthoré $\mathrm{S}$, Bourdès $\mathrm{V}$, Mercenier $\mathrm{A}$, Haddar $\mathrm{CH}$, Verhoeven PO, Andres P. Effect of a lotion containing the heat-treated probiotic strain Lactobacillus johnsonii NCC 533 on Staphylococcus aureus colonization in atopic dermatitis. Clin Cosmet Investig Dermatol. 2017;10:249-57. https://doi.org/ 10.2147/CCID.S135529.

24. Myles IA, Earland NJ, Anderson ED, et al. First-inhuman topical microbiome transplantation with Roseomonas mucosa for atopic dermatitis. JCI Insight. 2018;3(9):e120608. https://doi.org/10. 1172/jci.insight.120608.

25. Sharma D, Kober MM, Bowe WP. Anti-aging effects of probiotics. J Drugs Dermatol. 2016;15(1):9-12.

26. Kang YM, Hong $\mathrm{CH}$, Kang $\mathrm{SH}$, et al. Anti-photoaging effect of plant extract fermented with Lactobacillus buchneri on CCD-986sk fibroblasts and HaCaT keratinocytes. J Funct Biomater. 2020;11(1): 3. https://doi.org/10.3390/jfb11010003.

27. Notay M, Saric-Bosanac S, Vaughn AR, Dhaliwal S, Trivedi M, Reiter PN, Rybak I, Li CC, Weiss LB, Ambrogio L, Burney W, Sivamani RK. The use of topical Nitrosomonas eutropha for cosmetic improvement of facial wrinkles. J Cosmet Dermatol. 2020;19(3):689-93. https://doi.org/10.1111/ jocd. 13060 .
28. Benhadou F, Mintoff D, Schnebert B, Thio HB. Psoriasis and microbiota: a systematic review. Diseases. 2018;6(2):47. https://doi.org/10.3390/ diseases6020047.

29. Chang H, Yan D, Singh R, et al. Alteration of the cutaneous microbiome in psoriasis and potential role in Th17 polarization. Microbiome. 2018;6:154. https://doi.org/10.1186/s40168-018-0533-1.

30. Gao Z, Tseng CH, Strober BE, Pei Z, Blaser MJ. Substantial alterations of the cutaneous bacterial biota in psoriatic lesions. PLoS ONE. 2008;3(7): e2719. https://doi.org/10.1371/journal.pone. 0002719 .

31. Damiani G, Bragazzi NL, McCormick TS, et al. Gut microbiota and nutrient interactions with skin in psoriasis: a comprehensive review of animal and human studies. World J Clin Cases. 2020;8(6): 1002-12. https://doi.org/10.12998/wjcc.v8.i6.1002.

32. Yu Y, Dunaway S, Champer J, Kim J, Alikhan A. Changing our microbiome: probiotics in dermatology. Br J Dermatol. 2020;182(1):39-46. https:// doi.org/10.1111/bjd.18088.

33. Knackstedt R, Knackstedt T, Gatherwright J. The role of topical probiotics on wound healing: a review of animal and human studies. Int Wound $\mathrm{J}$. 2020. https://doi.org/10.1111/iwj.13451.

34. Valdéz JC, Peral MC, Rachid M, Santana M, Perdigón G. Interference of Lactobacillus plantarum with Pseudomonas aeruginosa in vitro and in infected burns: the potential use of probiotics in wound treatment. Clin Microbiol Infect. 2005;11(6):472-9. https://doi.org/10.1111/j.1469-0691.2005.01142.x.

35. Peral MC, Huaman Martinez MA, Valdez JC. Bacteriotherapy with Lactobacillus plantaruminburns. Int Wound J. 2009;6(1):73-81. https://doi.org/10. 1111/j.1742-481x.2008.00577.x.

36. Peral MC, Rachid MM, Gobbato NM, Martinez $\mathrm{MAH}$, Valdez JC. Interleukin-8 production by polymorphonuclear leukocytes from patients with chronic infected leg ulcers treated with Lactobacillus plantarum. Clin Microbiol Infect. 2010;16(3):281-6. https://doi.org/10.1111/j.1469-0691.2009.02793.x. 